\title{
A NOTE ON VERISIMILITUDE AND ACCURACY
}

\author{
RANDALL G. MCCUTCHEON
}

\begin{abstract}
Schoenfield has constructed examples of proper inaccuracy measures that value verisimilitude (in a certain sense) in spaces of worlds equipped with a particular variety of verisimilitude metric. However, Schoenfield left it as an open question whether 'for every space of worlds, there is a proper inaccuracy measure that values verisimilitude.' Here we answer this question in the affirmative.
\end{abstract}

Let $\Omega=\left\{\omega_{1}, \ldots, \omega_{n}\right\}$ be a set of worlds. We represent credence functions on $\Omega$ as $n$-tuples $a=\left(a_{1}, \ldots, a_{n}\right)$, where $a_{i} \geq 0$ and $a_{1}+\cdots+a_{n}=1$. $\left(a_{i}\right.$ represents credence in $\left\{\omega_{i}\right\}$.) An inaccuracy measure on $\Omega$ is a realvalued function $I$ giving, for each pair $(a, \omega)$, where $a$ is a credence function and $\omega \in \Omega$, the 'inaccuracy' $I(a, \omega)$ of $a$ should $\omega$ turn out to be actual. $I$ is proper if it has the property that the expected inaccuracy of $a$ is minimized for $a$ equal to the actual chances, and strictly proper if the minimum is uniquely realized at the actual chances.

It is usually supposed that inaccuracy is blind to similarities between the worlds, and is in fact a function of (1) the credence one assigns to the actual world, and (2) the distribution of credences one assigns to the non-actual worlds. If $I$ is that sort of inaccuracy measure then $\left(\frac{1}{2}, 0, \frac{1}{2}\right)$ is no more inaccurate than $\left(\frac{1}{2}, \frac{1}{2}, 0\right)$ when $\omega_{1}$ is actual, even if $\omega_{3}$ is less similar to $\omega_{1}$ (according to a similarity measure) than is $\omega_{2}$.

A contrasting view values 'truthlikeness'. It says that investing relatively greater credence in non-actual worlds that are more similar to the actual one is an epistemic good that should be reflected in our measures of inaccuracy. Aiming to demonstrate the costs of the truthlikeness view, Oddie ([2017]) shows that a certain principle that is 'universally embraced within the truthlikeness programme turns out to be incompatible with [...] propriety'. This principle, which Oddie terms 'Weak Proximity', requires that inaccuracy doesn't increase when one replaces a credence function supported on a set $E$ of worlds by a credence function concentrated on an $E$-world closest to the actual world.

Schoenfield ([2019]) counters that a truthlikeness view needn't be committed to anything so strong as Weak Proximity, arguing instead that the commitment is to employ inaccuracy measures according to which (in a certain sense) 'holding all else fixed, the more verisimilar 
credence function is more accurate.' Schoenfield thus replaces Weak Proximity with a new truthlikeness relation (Proximity 1) satisfied by certain triples $(I, \Omega, d)$, where $\Omega$ is a set of worlds, $d$ a certain variety of 'disagreement metric' on $\Omega$ and $I$ a proper inaccuracy measure, and gives non-trivial examples of triples satisfying this relation.

However, Schoenfield concedes that 'Since...my proofs will make use of particular spaces of worlds, I will not have shown that for every space of worlds, there is a proper inaccuracy measure that values verisimilitude'. Here we shall answer (in the affirmative) the question implicit in this passage. Specifically, we show that for arbitrary $(\Omega, d)$ (typically $d$ will be a metric on $\Omega$, though all we assume is that $d(x, x)=0$ and $d(x, y)=d(y, x)>0$ when $x \neq y)$ there exists a (strictly) proper inaccuracy measure $I$ that respects verisimilitude (in a certain sense).

Our first step is to define the relation that will play the role here that Proximity 1 plays in Schoenfield's work.

Proxvexity: Consider a finite set of worlds $\Omega$ equipped with a distance measure $d$ and let $I$ be an inaccuracy measure on credence functions over $\Omega$. Then $(\Omega, d, I)$ satisfies the Proxvexity relation if $I$ judges $b$ to be strictly more accurate than $c$ at $w_{a} \in \Omega$ whenever $b\left(\left\{\omega_{a}\right\}\right)>0$ and there is a partition $\mathcal{P}$ of $\Omega$ such that the following conditions hold:

(a) Every cell of $\mathcal{P}$ has either one or two members, with at least one cell having two.

(b) If $\{\omega\}$ is a singleton member of $\mathcal{P}$ then $b(\{\omega\})=c(\{\omega\})$.

(c) If $\left\{\omega, \omega^{*}\right\}$ is a 2-element member of $\mathcal{P}$ with $d\left(\omega_{a}, \omega\right) \leq d\left(\omega_{a}, \omega^{*}\right)$ then $c\left(\left\{\omega, \omega^{*}\right\}\right)=b\left(\left\{\omega, \omega^{*}\right\}\right)$ and $c(\{\omega\})<b(\{\omega\}) \leq c\left(\left\{\omega^{*}\right\}\right){ }^{1}$

We remark that, apart from being more general, Proxvexity guarantees stronger truthlikeness properties than Proximity 1 even within the latter's domain of application. (In particular it requires, for the relevant spaces, no restriction to partitions whose 2 member cells are such that 'the further of the two worlds...from $w_{a}$ disagrees with $w_{a}$ about all the atomic propositions that the closer of the two worlds disagrees with $w_{a}$ about [...]': see Schoenfield 2019.) Also, the inaccuracy measures Schoenfield constructs are not (except in some artificial cases) strictly proper. So the following result is novel in several respects.

\footnotetext{
${ }^{1}$ Note: the latter inequality is strict if $d\left(\omega_{a}, \omega\right)=d\left(\omega_{a}, \omega^{*}\right)$. (To see this, just switch the roles of $w$ and $w^{*}$.)
} 


\section{Theorem}

For any finite set $\Omega$ of worlds that is equipped with a distance measure $d$, there exists a strictly proper inaccuracy measure $I$ such that $(\Omega, d, I)$ satisfies the Proxvexity relation.

\section{Proof}

Since the theorem is trivial for spaces of fewer than three worlds, we shall assume that $\Omega$ has at least three elements.

Let $f:(0, \infty) \rightarrow(0, \infty)$ be any strictly decreasing function, for example $f(x)=\frac{1}{1+x}$. Define

$$
I\left(\left(a_{1}, \ldots, a_{n}\right), \omega_{k}\right)=\sum_{1 \leq i<j \leq n} f\left(d\left(\omega_{i}, \omega_{j}\right)\right) L\left(\left(a_{1}, \ldots, a_{n}\right), \mathcal{Q}_{i j}, \omega_{k}\right) .
$$

Here $\mathcal{Q}_{i j}=\left\{\left\{\omega_{i}, \omega_{j}\right\}\right\} \cup\left\{\left\{\omega_{t}\right\}: t \notin\{i, j\}\right\}$ and

$$
L\left(\left(a_{1}, \ldots, a_{n}\right), \mathcal{Q}, \omega_{k}\right)=-\log \left(\sum_{i \in C} a_{i}\right),
$$

where $\left\{\omega_{i}: i \in C\right\}$ is the cell of $\mathcal{Q}$ containing $\omega_{k}$.

$L\left(\left(a_{1}, \ldots, a_{n}\right), \mathcal{Q}, \omega_{k}\right)$ is the inaccuracy of an agent having credence function $\left(a_{1}, \ldots, a_{n}\right)$ over $\left(\omega_{1}, \ldots, \omega_{n}\right)$ when $w_{k}$ is actual and the agent is scored over the partition $\mathcal{Q}$ by the logarithmic inaccuracy measure, which is strictly proper (see Good 1952). So, since any linear combination of proper inaccuracy measures with non-negative weights is itself a proper inaccuracy measure, $I$ is proper. In fact $I$ is strictly proper, for if $a_{k}$ differs from the actual probability then $L\left(\left(a_{1}, \ldots, a_{n}\right), \mathcal{Q}_{i j}, \omega_{k}\right)$ has strictly greater than minimal expectation when $k \notin\{i, j\}$.

Finally we show that $(\Omega, d, I)$ satisfies the Proxvexity relation. Suppose to this end that $b=\left(b_{1}, \ldots, b_{n}\right)$ and $c=\left(c_{1}, \ldots, c_{n}\right)$ are credence functions over $\Omega$ and there are $\omega_{a} \in \Omega$ and a partition $\mathcal{P}$ of $\Omega$ such that (a), (b) and (c) are satisfied. (See above.) We will show that if $b\left(\left\{\omega_{a}\right\}\right)>0$ then $I$ judges $b$ to be strictly more accurate than $c$ at $\omega_{a}$.

By induction, it suffices to consider the case where $\mathcal{P}$ has exactly one cell $\left\{w, w^{*}\right\}$ having two members. If one of the members of this two member cell is the actual world $w_{a}$ the result follows immediately, as in this case $b\left(\left\{\omega_{a}\right\}\right)>c\left(\left\{\omega_{a}\right\}\right)$ and

$$
L\left(\left(b_{1}, \ldots, b_{n}\right), \mathcal{Q}_{i j}, \omega_{a}\right) \leq L\left(\left(c_{1}, \ldots, c_{n}\right), \mathcal{Q}_{i j}, \omega_{a}\right)
$$

for every $1 \leq i<j \leq n$, with equality only when $\left\{\omega_{i}, \omega_{j}\right\}=\left\{\omega, \omega^{*}\right\}$.

We may therefore assume without loss of generality that $\omega_{a}=\omega_{1}$, $\omega=\omega_{2}$ and $\omega^{*}=\omega_{3}$, with $d\left(\omega_{1}, \omega_{2}\right) \leq d\left(\omega_{1}, \omega_{3}\right)$. Note that $b_{1}=c_{1}=$ $r>0, b_{2}+b_{3}=c_{2}+c_{3}, f\left(d\left(\omega_{1}, \omega_{2}\right)\right) \geq f\left(d\left(\omega_{1}, \omega_{3}\right)\right)$ and $c_{2}<b_{2} \leq c_{3}$, with $b_{2}<c_{3}$ if $f\left(d\left(\omega_{1}, \omega_{2}\right)\right)=f\left(d\left(\omega_{1}, \omega_{3}\right)\right)$. Then 


$$
\begin{aligned}
& I\left(\left(b_{1}, \ldots, b_{n}\right), \omega_{a}\right)-I\left(\left(c_{1}, \ldots, c_{n}\right), \omega_{a}\right) \\
= & f\left(d\left(\omega_{1}, \omega_{2}\right)\right)\left(-\log \left(b_{1}+b_{2}\right)+\log \left(c_{1}+c_{2}\right)\right) \\
& +f\left(d\left(\omega_{1}, \omega_{3}\right)\right)\left(-\log \left(b_{1}+b_{3}\right)+\log \left(c_{1}+c_{3}\right)\right) \\
= & f\left(d\left(\omega_{1}, \omega_{2}\right)\right) \log \frac{r+c_{2}}{r+b_{2}}+f\left(d\left(\omega_{1}, \omega_{3}\right)\right) \log \frac{r+c_{3}}{r+b_{3}} \\
\leq & f\left(d\left(\omega_{1}, \omega_{3}\right)\right) \log \frac{r+c_{2}}{r+b_{2}}+f\left(d\left(\omega_{1}, \omega_{3}\right)\right) \log \frac{r+c_{3}}{r+b_{3}} \leq 0 .
\end{aligned}
$$

The final inequality is strict ${ }^{2}$ if $b_{2}<c_{3}$, and the penultimate inequality is strict if $f\left(d\left(\omega_{1}, \omega_{2}\right)\right)>f\left(d\left(\omega_{1}, \omega_{3}\right)\right)$. Since either $b_{2}<c_{3}$ or $f\left(d\left(\omega_{1}, \omega_{2}\right)\right)>f\left(d\left(\omega_{1}, \omega_{3}\right)\right)$ must hold, we are done.

We conclude with the following question, which asks whether the inaccuracy measure guaranteed by the above Theorem can be chosen bounded.

\section{Question}

Is it the case that for any finite set $\Omega$ of worlds equipped with a distance measure $d$, there exists a strictly proper, bounded inaccuracy measure $I$ such that $(\Omega, d, I)$ satisfies the Proxvexity relation?

\section{Acknowledgements}

Thanks to Miriam Schoenfield, the philosophy department at Ohio State, two anonymous reviewers and the editors of BJPS.

Randall G. McCutcheon

Department of Mathematical Sciences University of Memphis, Memphis, TN U.S.A.

rmcctchn@memphis.edu

\section{References}

Good, I.J. [1952]: 'Rational Decisions', Journal of the Royal Statistical Society, Ser. B, 14, pp. 107-114.

Oddie, G. [2019]: 'What Accuracy Could Not Be', British Journal for the Philosophy of Science, 70, pp. 551-580.

Schoenfield, M. [2019]: 'Accuracy and Verisimilitude: the Good, the Bad and the Ugly', British Journal for the Philosophy of Science. Forthcoming.

\footnotetext{
${ }^{2}$ What must be shown in this case is that $\left(r+c_{2}\right)\left(r+c_{3}\right)<\left(r+b_{2}\right)\left(r+b_{3}\right)$, which after substitution of $b_{3}=c_{2}+c_{3}-b_{2}$, expansion and cancellation reduces to $\left(b_{2}-c_{2}\right)\left(b_{2}-c_{3}\right)<0$.
} 